“ (C) 2016 IEEE. Personal use of this material is permitted. Permission from IEEE must be obtained for all other uses, in any current or future media, including

reprinting/republishing this material for advertising or promotional purposes, creating new collective works, for resale or redistribution to servers or lists, or reuse of any copyrighted component of this work in other works." 


\title{
AC Small Signal Modeling of PWM Y-Source Converter by Circuit Averaging and Averaged Switch Modeling Technique
}

\author{
Mojtaba Forouzesh ${ }^{1,2}$, Yam P. Siwakoti ${ }^{2}$, Frede Blaabjerg ${ }^{2}$, Sara Hasanpour ${ }^{3}$ \\ ${ }^{1}$ Department of Electrical Engineering, University of Guilan, Rasht, Iran. \\ ${ }^{2}$ Department of Energy Technology, Aalborg University, Aalborg, Denmark. \\ ${ }^{3}$ Department of Electrical Engineering, Ramsar Branch, Islamic Azad University, Ramsar, Iran. \\ E-Mail: m.forouzesh.ir@ieee.org, yas@et.aau.dk, fbl@et.aau.dk, s.hasanpour@iauramsar.ac.ir
}

\section{Keywords}

«Impedance source network», «Converter control», «Small signal modeling», «Pulse Width Modulation (PWM)», «Magnetically coupled Y-source network», «Z-source converter».

\begin{abstract}
Magnetically coupled Y-source impedance network is a newly proposed structure with versatile features intended for various power converter applications e.g. in the renewable energy technologies. The voltage gain of the Y-source impedance network rises exponentially as a function of turns ratio, which is inherited from a special coupled inductor with three windings. Due to the importance of modeling in the converter design procedure, this paper is dedicated to dc and ac small signal modeling of the PWM Y-source converter. The derived transfer functions are presented in detail and have been verified through simulation and experimental results.
\end{abstract}

\section{Introduction}

Impedance sources have been originated in order to obviate some drawbacks related to the conventional voltage and current source converters. The first impedance network was called Z-source due to its schematic appearance. The main merits of Z-source impedance network can be named as simultaneous having voltage buck/boost ability, high immunity to miss gating of switches and improved reliability. Afterward, various Z-source based impedance networks have been presented for different converters (dc-dc, dc-ac, ac-dc or ac-ac) [1]. In recent years, a new trend has been initiated to achieve even higher voltage boost in impedance networks. Among various presented structures and techniques, the coupled inductor based impedance network due to its high voltage boosting ability proved to be of high interest. Some of the well-known Magnetically Coupled Impedance Sources (MCIS) can be named as T-source [2], trans-Z-source [3], $\Gamma$-source [4], and Y-source [5]. Among the magnetically coupled impedance sources, the Y-source network has special characteristics like a very high voltage gain with a small shoot-through time; in addition, it has more degrees of freedom for tuning its gain, and hence, more design freedom. Recently, different structures of Y-source network have been implemented for realization of various converters [6]-[8].

For designing a reliable closed loop controller for impedance source based converters, the system dynamic model should be thoroughly investigated before the control design procedure. Typically, the small signal modeling of impedance source based converters solely contribute to the impedance network and not to other additional parts in different converters [9]. Various small signal modeling of the Z-source converter can be found in the literature. In [10], a signal flow graph modeling of switched $Z$-source converter has been investigated. A state space analysis has been used for small signal modeling of Z-source and quasi-Z-source converters in [11] and [12], respectively. In [13], a small signal modeling of the pulse width modulated Z-source converter is presented by circuit averaging technique. In general, only a few papers can be found in the literature regarding the modeling of the converters with magnetic components. Indeed, this paper addresses the small signal modeling of the PWM Y-source converter with three coupled inductors.

Since there are various non-linear elements like MOSFET or diode in the circuit of power electronics converters, they should be linearized in order to conduct liner control theory. There are two main 
methods for circuit linearization, state space averaging and circuit averaging techniques. The average values of capacitor voltage and inductor current are of interest for power circuit designers, the circuit averaging method seems to be more interesting for linearization of PWM converters [14], [15]. Hence, in this paper the derivation of averaged dc model and ac small signal model of the PWM Y-source converter is presented by means of circuit averaging and averaged switch technique. Subsequently two transfer functions for input to capacitor voltage and duty cycle to capacitor voltage are derived, which corresponds to disturbances in the input voltage and the duty cycle, respectively.

\section{Derivation of The Small Signal Model for The PWM Y-source Converter}

Fig. 1 shows the PWM Y-source converter with perturbation of input voltage $\left(v_{i n}\right)$, duty cycle $(D)$ and load current $\left(i_{o}\right)$. In Fig. 1, the symbol $\simeq$ stands for average value (dc part) plus perturbation (ac part). There are some predominant notations in the following of this paper, namely $v_{\text {in }}=V_{I N}+\hat{v}_{\text {in }}, i_{\text {in }}=$ $I_{I N}+\hat{\imath}_{i n}, v_{o}=V_{O}+\hat{v}_{o}, i_{o}=I_{O}+\hat{\imath}_{o}$ and $d=D+\hat{d}$. The capital quantities are averaged values and quantities with "hat" are perturbation values. Apparently, quantities with "hat" are time variant values that lead to overall time variant quantities, in order to simplify notations, subscript " $(t)$ " are neglected for all time variant quantities.

Following assumptions are considered for the small signal modeling and transfer function derivations. 1) PWM Y-source converter is operating in CCM. 2) All passive components are lossless and coupling coefficient of the coupled inductors are perfect (this is quite reasonable since Y-source network normally demands tightly coupled magnetics [5]). 3) All semiconductor switches, Diode and MOSFET are ideal. 4) The natural time constant of PWM Y-source converter is much longer than one switching period.

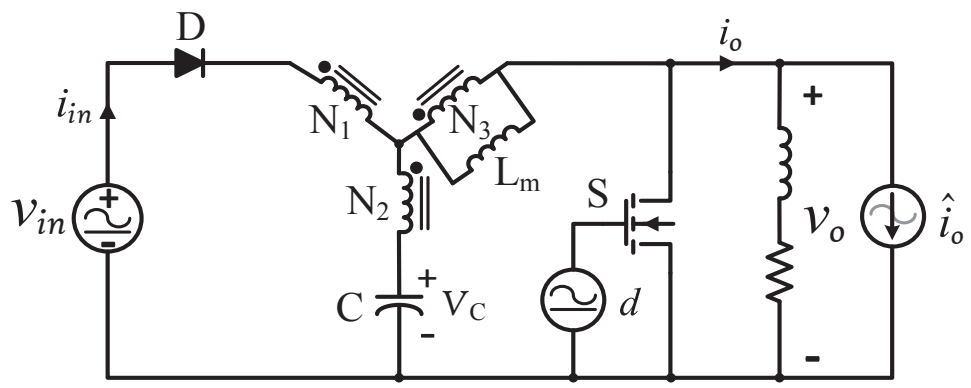

Fig. 1. PWM Y-source converter with variation of input voltage $\left(v_{i n}\right)$, duty cycle $(d)$ and load current $\left(i_{o}\right)$.

The main nonlinear components in the PWM Y-source converter are diode D and MOSFET S; other components like capacitor, coupled inductors and resistor are linear and time invariant. The first step in the switch PWM modeling approach is to pull out the switching components from the other circuit elements. Then, an average circuit model can be found in order to linearize the switching network. Fig. 2 shows the PWM Y-source converter with extracted two port switch network from the circuit. As shown in Fig. 2, terminal quantities at the two side of the switch network are $v_{1}(t), i_{1}(t), v_{2}(t)$ and $i_{2}(t)$. At each side, one quantity can be selected as an independent input and hence the two other quantities would be dependent inputs. However, the selection of independent quantities are arbitrary. It is typically common to select the current of diode and the voltage of MOSFET as independent quantities. Besides, the duty cycle $d$ would be as the independent control input. As it can be found from Fig. 3, four terminal voltages and currents of the PWM Y-source converter are as $v_{1}(t)=v_{D}$, $i_{1}(t)=i_{\text {in }}, v_{2}(t)=v_{O}$ and $i_{2}(t)=i_{S}$. As defined before, $i_{1}(t)$ and $v_{2}(t)$ are independent quantities, the next step is to find the dependent quantities $v_{1}(t)$ and $i_{2}(t)$ as functions of independents quantities [14]. Consequently, $v_{D}$ and $i_{S}$ are dependent values of the switch network that should be written solely based on dependent values $v_{O}$ and $i_{\text {in }}$.

In the PWM Y-source converter, the average value of output voltage is equal to the average value of the capacitor voltage. Hence, according to the steady state analysis of $Y$-source network, assuming the voltage across $L_{3}$ is $V_{L}$, the voltage of capacitor $\mathrm{C}$ can be obtained by applying volt-second balance principle to the inductor voltage $V_{L}[5]$. 


$$
V_{C}=V_{O}=\frac{1-D}{1-K D} V_{I N}
$$

In (1), $D$ is the duty cycle of shoot-through mode and $K$ is the winding factor of Y-source impedance network which can be defined as $K=\left(N_{3}+N_{1}\right) /\left(N_{3}-N_{2}\right)$. For a lossless circuit $\left(V_{\text {in }} I_{\text {in }}=V_{O} I_{O}\right)$, according to (1), the averaged output current of the PWM Y-source converter can be obtained.

$$
I_{O}=\frac{1-K D}{1-D} I_{I N}
$$

Considering (1) and (2), by using Kirchhoff's Voltage Law (KVL) and Current Law (KCL) in both shoot-through and non-shoot-through modes, the averaged values of the voltage across diode $\mathrm{D}$ and the current that pass through MOSFET $S$ can be obtained as follows.

$$
\begin{aligned}
& V_{D}=K^{\prime} \frac{D}{D^{\prime}} V_{O} \\
& I_{S}=K^{\prime} \frac{D}{D^{\prime}} I_{I N}
\end{aligned}
$$

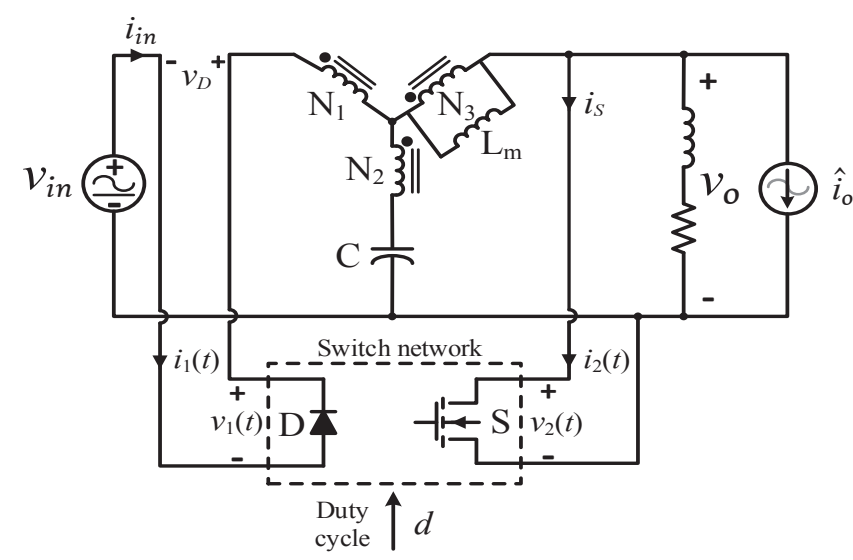

Fig. 2. PWM Y-source converter with illustration of the nonlinear switch network.

In (3) and (4), $D^{\prime}$ is the duty cycle of non-shoot-through mode and defined as $D^{\prime}=1-D ; K^{\prime}$ is the winding coefficient of the Y-source which introduced to simplify the following derivations and can be defined as $K^{\prime}=\left(N_{1}+N_{2}\right) /\left(N_{3}-N_{2}\right)$. Equations (3) and (4) represent the dc model of switch network. By substituting with time variant values and using approximation, the ac large signal model of the switch network can be written as follows.

$$
\begin{aligned}
& v_{D} \approx K^{\prime} \frac{d}{d \prime} v_{O} \\
& i_{S} \approx K^{\prime} \frac{d}{d \prime} i_{i n}
\end{aligned}
$$

In (5) and (6), $v_{D}$ and $i_{S}$ are large signal time variant quantities that are defined as $v_{D}=V_{D}+\hat{v}_{D}$ and $i_{S}=I_{S}+\hat{\imath}_{S}$. Considering small signal ac components for all quantities in (5) and (6), we have

$$
\begin{aligned}
& v_{D}=V_{D}+\hat{v}_{D}=K^{\prime} \frac{D+\hat{d}}{D^{\prime}-\hat{d}}\left(V_{O}+\hat{v}_{O}\right) \\
& i_{S}=I_{S}+\hat{\imath}_{S}=K^{\prime} \frac{D+\hat{d}}{D^{\prime}-\hat{d}}\left(I_{I N}+\hat{\imath}_{i n}\right)
\end{aligned}
$$

Expanding the second term in (7) and (8), and using the first order approximation for McLaurin series [11], the following equation is achieved.

$$
\frac{D+\hat{d}}{D^{\prime}-\hat{d}}=\frac{D+\widehat{d}}{D^{\prime}} \frac{1}{1-\frac{\widehat{d}}{D^{\prime}}}=\frac{D+\hat{d}}{D^{\prime}}\left(1+\frac{\hat{d}}{D^{\prime}}\right)
$$


Substituting (9) in (7) and (8), we have

$$
\begin{aligned}
& v_{D}=K^{\prime} \frac{D+\hat{d}}{D^{\prime}}\left(1+\frac{\hat{d}}{D^{\prime}}\right)\left(V_{O}+\hat{v}_{O}\right) \\
& i_{S}=K^{\prime} \frac{D+\hat{d}}{D^{\prime}}\left(1+\frac{\hat{d}}{D^{\prime}}\right)\left(I_{I N}+\hat{\imath}_{i n}\right)
\end{aligned}
$$

Expanding (10) and (11), and neglecting the terms with multiplication of two perturbation signals, lead to both a dc and ac small signal model of the switch network of the PWM Y-source converter.

$$
\begin{aligned}
& v_{D}=K^{\prime} \frac{D}{D \prime} V_{O}+K^{\prime} \frac{D}{D \prime} \hat{v}_{O}+K^{\prime} \frac{V_{O}}{D^{\prime 2}} \hat{d} \\
& i_{S}=K^{\prime} \frac{D}{D^{\prime}} I_{i n}+K^{\prime} \frac{D}{D^{\prime}} \hat{l}_{i n}+K^{\prime} \frac{I_{I N}}{D^{\prime 2}} \hat{d}
\end{aligned}
$$

The first terms in (12) and (13) represent the dc model and the second and third terms in (12) and (13) represent ac small signal linear model of diode D and MOSFET $S$. Linearization is realized by omitting second order terms of ac perturbed signals in (10) and (11). Now, the dc and ac small signal model of the PWM Y-source converter can be realized using voltage and current controlled sources. Fig. 3(a) illustrates the dc and linear small signal model of the PWM Y-source converter. Furthermore, the dc model and ac model of the PWM Y-source converter are illustrated in Fig. 3(b) and Fig. 3(c), respectively.

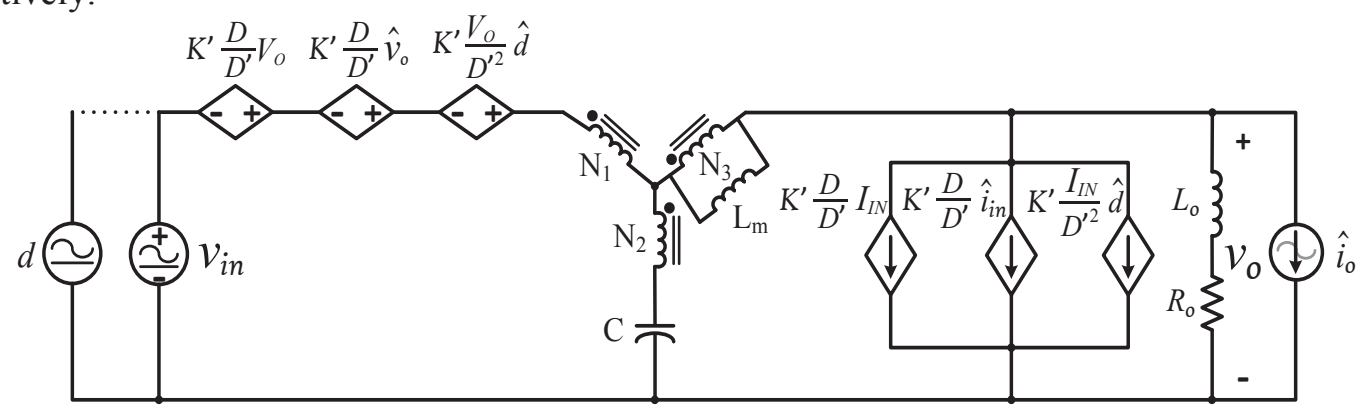

(a)

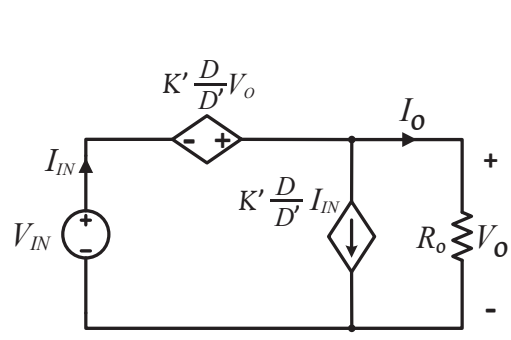

(b)

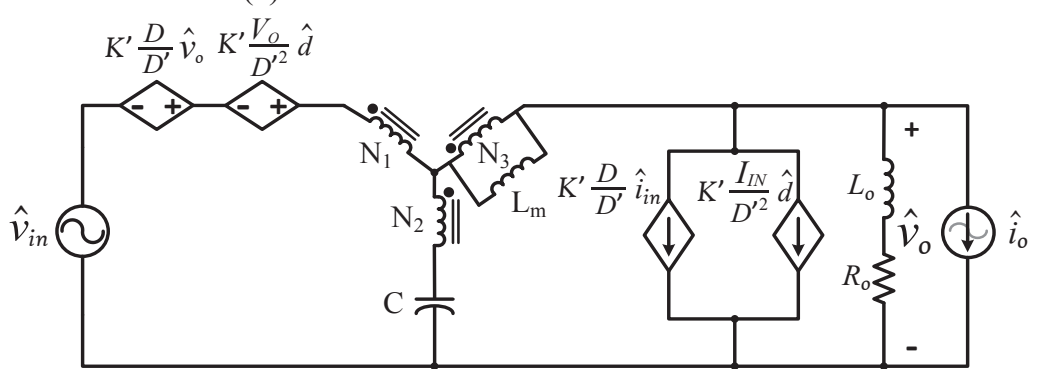

(c)

Fig. 3. (a) Dc and ac small signal model of PWM Y-source converter, (b) structure of dc model (c) structure of AC small signal model.

\section{Small Signal Equivalent Circuits and Derivation of Transfer Functions}

In this section, input to capacitor voltage and control to capacitor voltage transfer functions of the PWM Y-source converter are presented using the derived small signal model in the previous section.

\section{Input voltage to Capacitor Voltage Transfer Function}

In order to derive the transfer function from input voltage, the perturbation $\hat{d}$ must be ignored in the ac small signal model of PWM Y-source converter. In addition, for the following derivation in this section it is assumed that there is no perturbation in load current. Hence, letting $\hat{d}=0$ and $\hat{\imath}_{o}=0$ in Fig. 3(c), the ac small signal model to derive the input voltage to capacitor voltage transfer function 
can be achieved as shown in Fig. 4. The impedance of the coupled inductors are considered as a single impedance $\left(Z_{m}\right)$ on the third winding of PWM Y-source converter. The impedances of the ideal magnetizing inductor $\left(L_{m}\right)$ and the ideal capacitor $\mathrm{C}$ are expressed in (14) as

$$
Z_{m}=s L_{m}, Z_{C}=\frac{1}{s C}
$$

Moreover, in order to perform a practical analysis, after the transfer functions are derived in their impedance form, the ESRs of magnetizing inductor $\left(\mathrm{ESR}_{\mathrm{L}}\right)$ and capacitor $\mathrm{C}(\mathrm{ESR} \mathrm{c})$ can be added to their impedances. The load impedance is given in (15). It should be noted that the perturbation in the load current can be implemented through the load impedance $\left(Z_{o}\right)$.

$$
Z_{o}=R_{o}+s L_{o}
$$

As it is known, in a three winding coupled inductor, the following equation is dominant.

$$
N_{1} i_{L 1}+N_{2} i_{L 2}+N_{3} i_{L 3}=0
$$

From Fig. 4, applying KCL and using (16), the following equations can be written.

$$
\begin{aligned}
& \hat{\imath}_{\text {in }}=\frac{\hat{\imath}_{C}+\hat{\imath}_{O}}{1-K^{\prime} \frac{D}{D^{\prime}}} \\
& \hat{\imath}_{m}=\frac{N_{1}}{N_{3}} \hat{l}_{i n}+\frac{N_{2}}{N_{3}} \hat{l}_{C}+K^{\prime} \frac{D}{D^{\prime}} \hat{l}_{i n}+\hat{\imath}_{O}
\end{aligned}
$$

Applying KVL on the right and left loops of the circuit in Fig. 4, the following equations can be written.

$$
\begin{aligned}
& \hat{v}_{o}+\left(1-\frac{N_{2}}{N_{3}}\right) \hat{v}_{m}-\hat{v}_{C}=0 \\
& \hat{v}_{C}+\left(\frac{N_{1}+N_{2}}{N_{3}}\right) \hat{v}_{m}-\hat{v}_{i n}-K^{\prime} \frac{D}{D^{\prime}} \hat{v}_{o}=0
\end{aligned}
$$

Considering Ohm's law for the voltage across the magnetizing inductor, capacitor $\mathrm{C}$ and output voltage, and using (17) to (20), the input voltage to capacitor voltage transfer function $\left(G_{v v}\right)$ is derived in the impedance form as given by (21).

$$
G_{v v}=\frac{\hat{v}_{C}}{\hat{v}_{i n}}=\frac{D^{2} Z_{C} Z_{m} a_{1} a_{3}+D^{\prime}\left(D^{\prime}-K^{\prime} D\right) Z_{C} Z_{o}}{D^{\prime 2} Z_{C} Z_{m} a_{3}{ }^{2}+\left(D^{\prime}-K^{\prime} D\right)^{2} Z_{C} Z_{o}+Z_{m} Z_{o} a_{2}^{2}}
$$

In (21), $a_{1}, a_{2}$ and $a_{3}$ are the coefficients that are considered in order to simplify the transfer functions and defined as follows

$$
a_{1}=\frac{N_{3}-N_{2}}{N_{3}}, \quad a_{2}=\frac{N_{1}+N_{2}}{N_{3}}, \quad a_{3}=\frac{N_{1}+N_{3}}{N_{3}}
$$

Substituting (14)-(15) in (21), we get the s-domain transfer function of $G_{v v}$ as given by (22).

$$
G_{v v}=\frac{\hat{v}_{C}}{\hat{v}_{i n}}=\frac{\left[D^{2} L_{m} a_{1} a_{3}+D^{\prime}\left(D^{\prime}-K^{\prime} D\right) L_{o}\right] s+D^{\prime}\left(D^{\prime}-K^{\prime} D\right) R_{o}}{\left(C L_{m} L_{o} a_{2}^{2}\right) s^{3}+\left(C L_{m} R_{o} a_{2}{ }^{2}\right) s^{2}+\left[D^{\prime 2} L_{m} a_{3}^{2}+\left(D^{\prime}-K^{\prime} D\right)^{2} L_{o}\right] s+\left(D^{\prime}-K^{\prime} D\right)^{2} R_{o}}
$$

\section{Control to Capacitor Voltage Transfer Function}

In order to derive the transfer function from control perturbation $(\hat{d}), \hat{v}_{\text {in }}$ must be ignored in the ac small signal model of PWM Y-source converter. As mentioned before, no perturbation is considered in the load current. Hence, letting $\hat{v}_{i n}=0$ and $\hat{\imath}_{o}=0$ in Fig. 3(c), the ac small signal model to derive 
control to capacitor voltage transfer function can be achieved like shown in Fig. 5. Using (16) and KCL in Fig. 5, the following equations can be written.

$$
\begin{aligned}
& \hat{\imath}_{\text {in }}=\frac{\hat{\imath}_{C}+\hat{\imath}_{O}+K^{\prime} \frac{I_{I N} \prime^{2}}{1-K^{\prime}} \frac{D}{D^{\prime}}}{\hat{\imath}_{m}}=\frac{N_{1}}{N_{3}} \hat{\imath}_{i n}+\frac{N_{2}}{N_{3}} \hat{\imath}_{C}+K^{\prime} \frac{D}{D^{\prime}} \hat{\imath}_{i n}+K^{\prime} \frac{I_{I N}}{D^{\prime 2}} \hat{d}+\hat{\imath}_{O}
\end{aligned}
$$

Applying KVL on the left loop of the circuit in Fig. 5, the following equations can be written.

$$
\hat{v}_{C}+\left(\frac{N_{1}+N_{2}}{N_{3}}\right) \hat{v}_{m}-K^{\prime} \frac{D}{D^{\prime}} \hat{v}_{o}-K^{\prime} \frac{V_{O}}{D^{\prime 2}} \hat{d}=0
$$

Considering Ohm's law for the voltage across magnetizing inductor $\left(L_{m}\right)$, capacitor $\mathrm{C}$ and output voltage $\left(v_{o}\right)$, and using (19) and (23) to (25), the control to capacitor voltage transfer function $\left(G_{v d}\right)$ can be derived in the impedance form where the simplified transfer function is given by (26).

$$
G_{v d}=\frac{\hat{v}_{C}}{\widehat{d}}=\frac{K^{\prime} Z_{C} V_{O}\left[\left(D^{\prime}-K^{\prime} D\right) Z_{o}+D^{\prime} Z_{m} a_{1} a_{3}\right]-Z_{C} Z_{m} Z_{o} I_{I N} a_{2}\left(D^{\prime} K a_{2}+D K^{\prime} a_{3}\right)}{{D^{\prime}}^{3} Z_{C} Z_{m} a_{3}^{2}+D^{\prime 2} Z_{m} Z_{o} a_{2}^{2}+D^{\prime}\left(D^{\prime}-K^{\prime} D\right)^{2} Z_{C} Z_{o}+D D^{\prime} Z_{m} Z_{o} K^{\prime} a_{1} a_{2}}
$$

Substituting (14)-(15) into (26), we get the s-domain transfer function of $G_{v d}$ as given by (27).

$$
G_{v d}=\frac{\hat{v}_{C}}{\widehat{d}}=
$$

$\frac{-\left[I_{I N} L_{m} L_{O} a_{2}\left(D K^{\prime} a_{3}+D^{\prime} K a_{2}\right)\right] s^{2}+\left[D^{\prime} K^{\prime} V_{O} L_{m} a_{1} a_{3}-I_{I N} L_{m} R_{O} a_{2}\left(D K^{\prime} a_{3}+D^{\prime} K a_{2}\right)+\left(D^{\prime}-K^{\prime} D\right) K^{\prime} V_{O} L_{O}\right] s+\left(D^{\prime}-K^{\prime} V_{O} D\right) K^{\prime} V_{O} R_{O}}{\left[D^{\prime} C L_{m} L_{o} a_{2}\left(D K^{\prime} a_{1}+D^{\prime} a_{2}\right)\right] s^{3}+\left[D \prime C L_{m} R_{O} a_{2}\left(D K^{\prime} a_{1}+D^{\prime} a_{2}\right)\right] s^{2}+\left[D^{\prime 3} L_{m} a_{3}{ }^{2}+D^{\prime}\left(D^{\prime}-K^{\prime} D\right)^{2} L_{o}\right] s+D^{\prime}\left(D^{\prime}-K^{\prime} V_{O} D\right)^{2} R_{O}}$

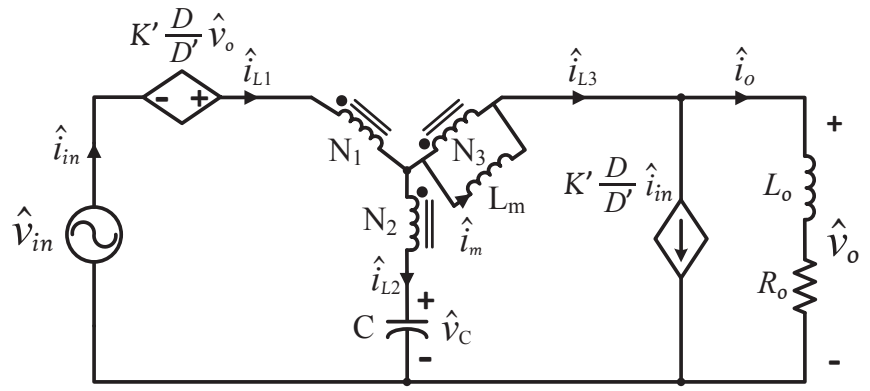

Fig. 4. Ac small signal model to derive input voltage to capacitor voltage transfer function.

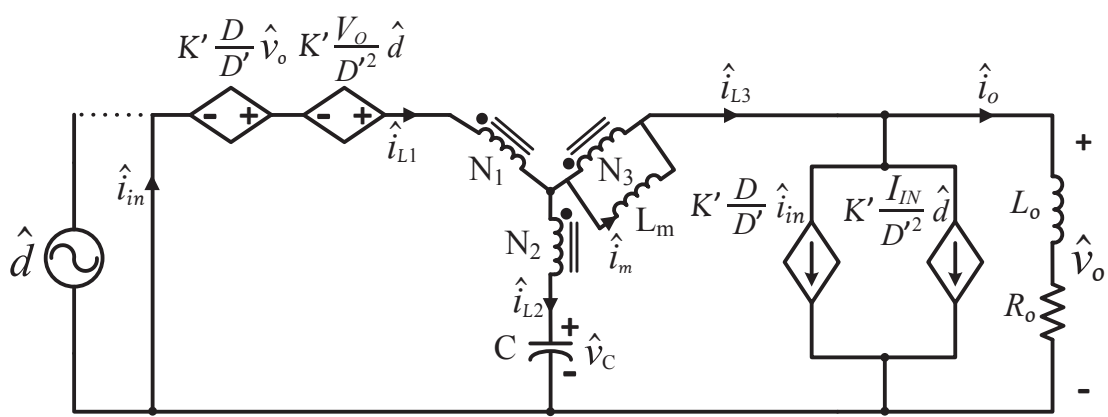

Fig. 5. Ac small signal model to derive control to capacitor voltage transfer function.

\section{Simulation and Experimental Results}

In order to validate the trueness of the derived transfer functions, some computer simulations have been done using MATLAB/Simulink software, demonstrating the dynamic and frequency responses of the PWM Y-source converter. The parameters used for computer simulations are as follows: $V_{I N}=15 \mathrm{~V}$, $D=0.1, f_{S}=25 \mathrm{kHz}, \mathrm{C}=470 \mu \mathrm{F}, L_{m}=1 \mathrm{mH}, \mathrm{N}_{1}: \mathrm{N}_{2}: \mathrm{N}_{3}=1: 2: 3, L_{o}=2.3 \mathrm{mH}, R_{O}=8 \Omega$. In order to verify the 
dynamic behavior of the derived model, the dynamic response of both detailed circuit and averaged large signal model to a step change in input voltage and duty cycle are depicted in Fig. 6(a) and Fig. 6(b), respectively. An unusual behavior can be seen in Fig. 6(b), where the capacitor voltage dips just before rising. This behavior is due to the right half plane zero in $G_{v d}$ which causes non-minimum phase effects [16]. In order to evaluate the frequency response, the detailed circuit of the PWM Ysource converter have been implemented in Simulink including the input voltage and duty cycle perturbation signals. A sinusoidal voltage signal with amplitude of $5 \mathrm{~V}$ and a sinusoidal reference wave with amplitude of $0.05 \mathrm{~V}$ have been injected to the input voltage source and gate of the MOSFET, respectively. Fig. 7(a) and Fig. 7(b) illustrate the frequency response from detailed switch circuit along with the predicted frequency response from transfer functions $G_{v v}$ and $G_{v d}$, respectively. Apparently, both dynamic and frequency responses are in good agreement with the dynamic and frequency responses from detailed PWM Y-source circuit. It should be noted that the parasitic resistances are ignored in the simulations.

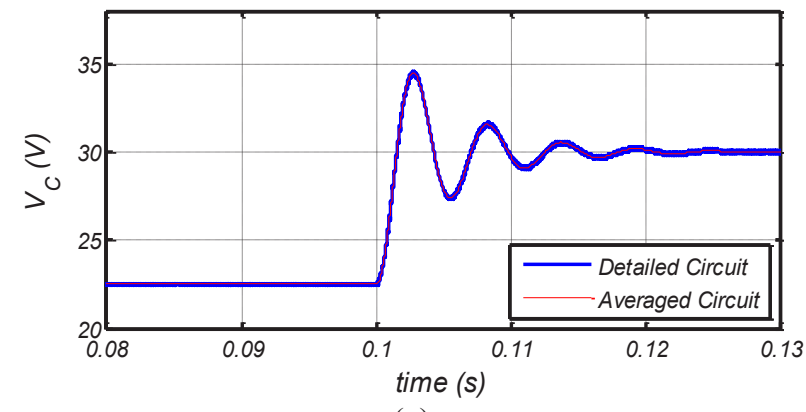

(a)

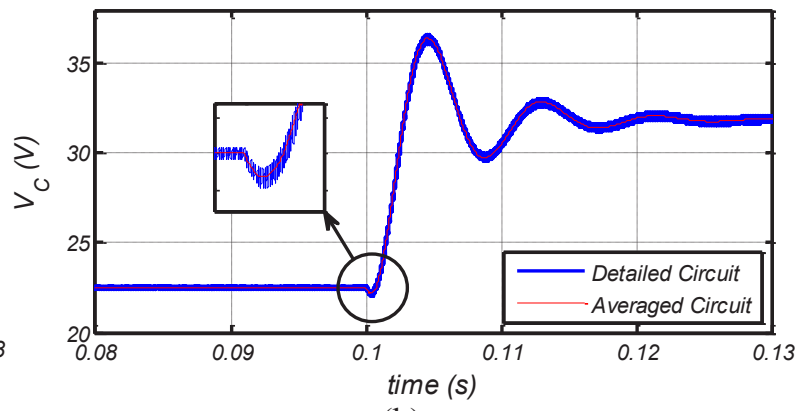

(b)

Fig. 6. Dynamic response of PWM Y-source converter to a step change in (a) input voltage from $15 \mathrm{~V}$ to $20 \mathrm{~V}$ and (b) duty cycle from 0.1 to 0.15 .

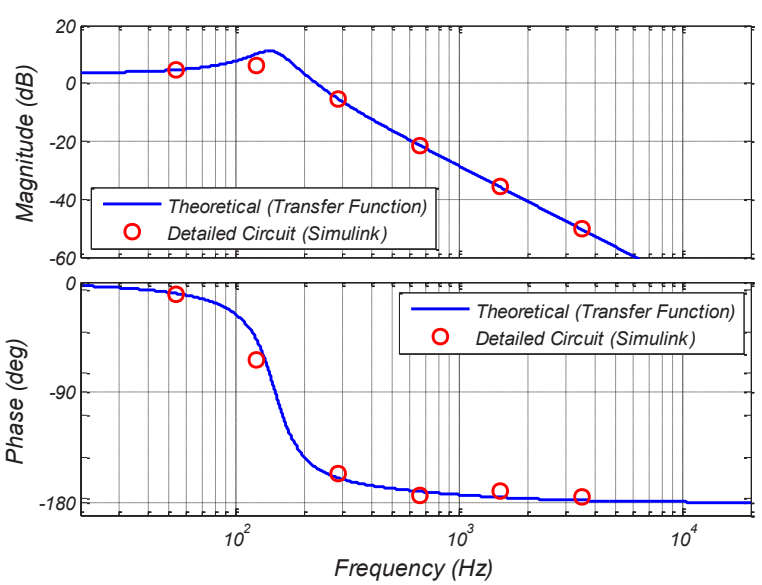

(a)

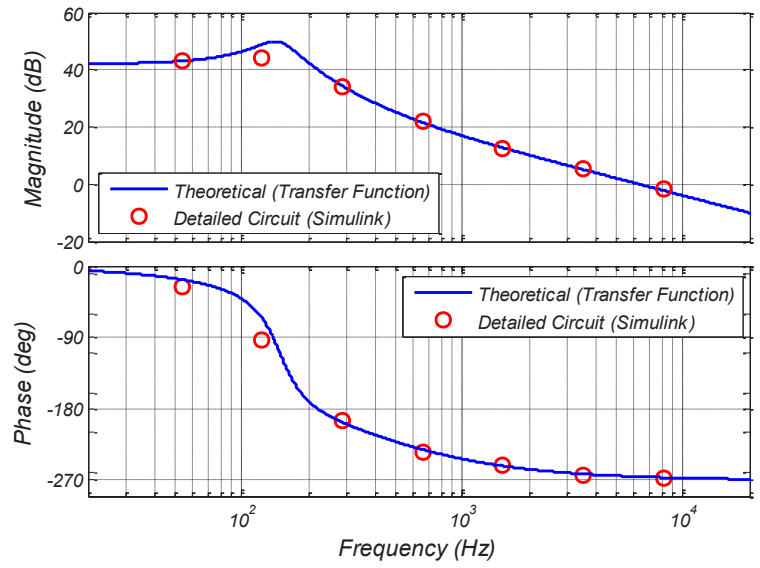

(b)

Fig. 7. Frequency response of PWM Y-source converter for (a) input to capacitor voltage $\left(G_{v v}\right)$ and (b) control to capacitor voltage $\left(G_{v d}\right)$.

In order to validate the effectiveness of the derived model and simulation results, some measurements have been done for the mentioned PWM converter. A prototype of the PWM Y-source converter has been designed and built in the laboratory. Detailed parameters used for the experiment are specified in Table I. The schematic drawing of the experimental setup to measure the frequency response is shown in Fig. 8(a). A Bode 100 vector network analyzer was used to measure the transfer function gain-phase of the PWM Y-source converter (bode plots). The frequency measurement of the derived transfer functions are comprised of two different phase, with perturbation in input voltage $\left(\hat{v}_{i n}\right)$ and with perturbation in the duty cycle $(\hat{d})$. In order to inject the ac small signal to the input voltage a J2120A transformer was employed as a line injector. An analogue modulator with Texas Instrument TLV3502 comparator was built to generate PWM for the switch. The ac small signal generated by Bode 100 for duty cycle is injected through a B-WIT 100 injection transformer and an injection resistor $\left(R_{\text {inj }}\right)$. 
Moreover, the Single Pole Double Throw (SPDT) switches (SPDT1 and SPDT2) define the appropriate connections of the measuring instrument to the Device Under Test (DUT) to measure the input to capacitor voltage $\left(G_{v v}\right)$ and control to capacitor voltage $\left(G_{v d}\right)$ transfer functions. A picture of the measurement setup implemented in the laboratory is shown in Fig. 8(b).

Table I: The parameters used for experiment of the PWM Y-source converter

\begin{tabular}{|l|l|c|}
\hline \multicolumn{2}{|c|}{ Parameter/Description } & Value \\
\hline Input voltage $\left(V_{I N}\right)$ & $15 \mathrm{~V}$ \\
\hline \multirow{2}{*}{ Load } & Resistance $\left(R_{o}\right)$ & $40 \Omega$ \\
\cline { 2 - 3 } & Inductance $\left(L_{o}\right)$ & $2.3 \mathrm{mH}$ \\
\hline \multirow{2}{*}{ Switching frequency $\left(f_{s}\right)$} & $25 \mathrm{kHz}$ \\
\hline Shoot-through duty cycle $(D)$ & $8 \%$ \\
\hline \multirow{2}{*}{ Capacitor C } & Capacitance & $870 \mu \mathrm{F}$ \\
\cline { 2 - 3 } & ESR & $15: 30: 45 \mathrm{~m} \Omega$ \\
\hline \multirow{2}{*}{ Turns Ratio } & Y-source network $\left(\mathrm{N}_{1}: \mathrm{N}_{2}: \mathrm{N}_{3}\right)$ & $(86: 334: 710) \mu \mathrm{H}$ \\
\hline \multirow{2}{*}{ Winding inductance } & Y-source network $\left(\mathrm{L}_{1}: \mathrm{L}_{2}: \mathrm{L}_{3}\right)$ & $(64: 230: 490) \mathrm{m} \Omega$ \\
\hline Winding resistance & Y-source network $\left(\mathrm{R}_{1}: \mathrm{R}_{2}: \mathrm{R}_{3}\right)$ & $\mathrm{C} 2 \mathrm{M} 0080120 \mathrm{D}$ \\
\hline Switch $S$ & $\mathrm{C} 3 \mathrm{D} 25170 \mathrm{H}$ \\
\hline Diode D & \\
\hline
\end{tabular}

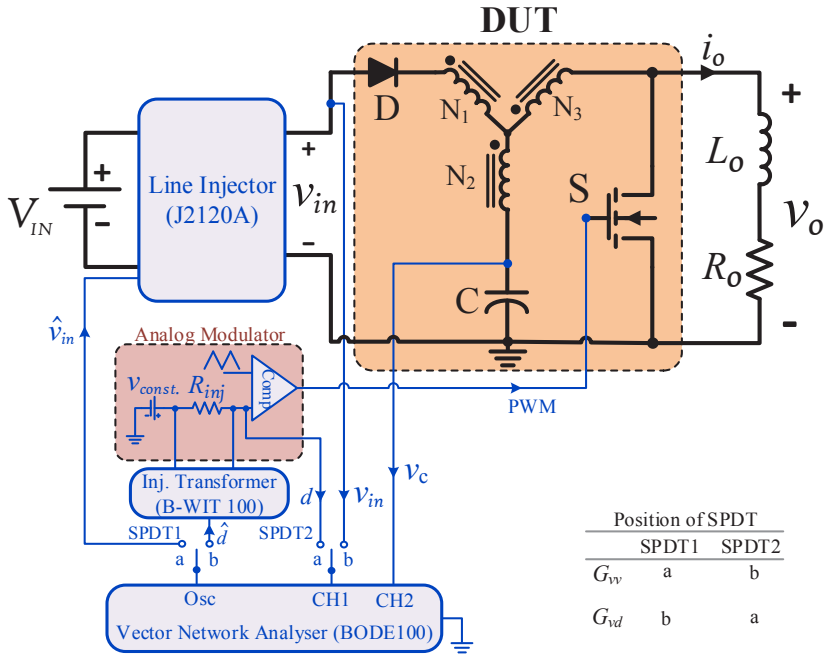

(a)

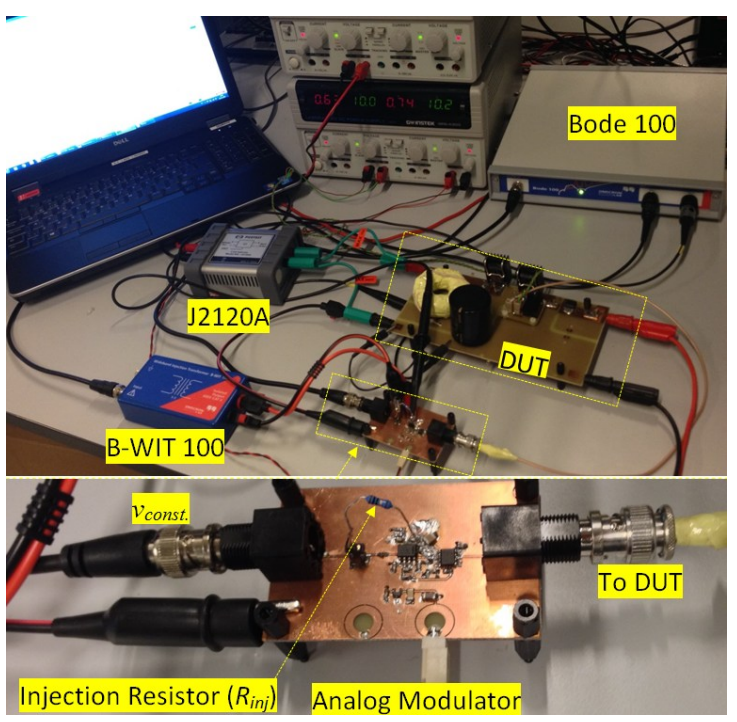

(b)

Fig. 8. Experimental setup, (a) schematic diagram to measure $G_{v v}$ and $G_{v d}$ of PWM Y-source converter and (b) picture of the experimental setup in the laboratory.

The measured frequency responses for PWM Y-source converter are shown in Fig. 9, in which the experimental results are shown in the left column and the theoretically predicted results from the derived transfer functions including the series resistances of inductor and capacitor are shown in the right column. In Fig. 9, TR1 represents the magnitude in decibel (dB) and TR2 represents the phase in degree; when the phase range in not between $\pm 180^{\circ}$, the "Unwrapped Phase" function is available in Bode 100 to display an unwrapped continuous reading of the phase. As it is clearly seen both experimental and simulation results are in good agreement. This convincing behavior for the system is due to the fact that the parasitic resistances are included in those derived transfer functions.

Fig. 10 illustrates the experimentally results along with the theoretically obtained dynamic response for PWM Y-source dc-dc converter to a step change in the input voltage from $15 \mathrm{~V}$ to $20 \mathrm{~V}$. Fig. 11 illustrates the experimentally along with the theoretically obtained dynamic response for PWM Ysource converter to a step change in duty cycle from 0.1 to 0.15 . The circuit parameters of the computer simulations are identical to the parameters of experimental setup. Moreover, to be compatible with the experimental setup, an $1.1 \Omega$ measured resistance from the input of the coupled 
inductors, which corresponds to loosely connected wires and connections of in the dynamic response, is also considered in the averaged model. In Fig. 11(a), a voltage dip can be seen just before the capacitor voltage starts to rise, which can be attributed to the non-minimum phase effect of the control to capacitor voltage transfer function. It should be mentioned that because of neglecting the switching power loss in the averaged circuit model there is a small difference between the experimental and simulation results, which due to the increased loss at high duty cycle this difference is more considerable in Fig. 11.
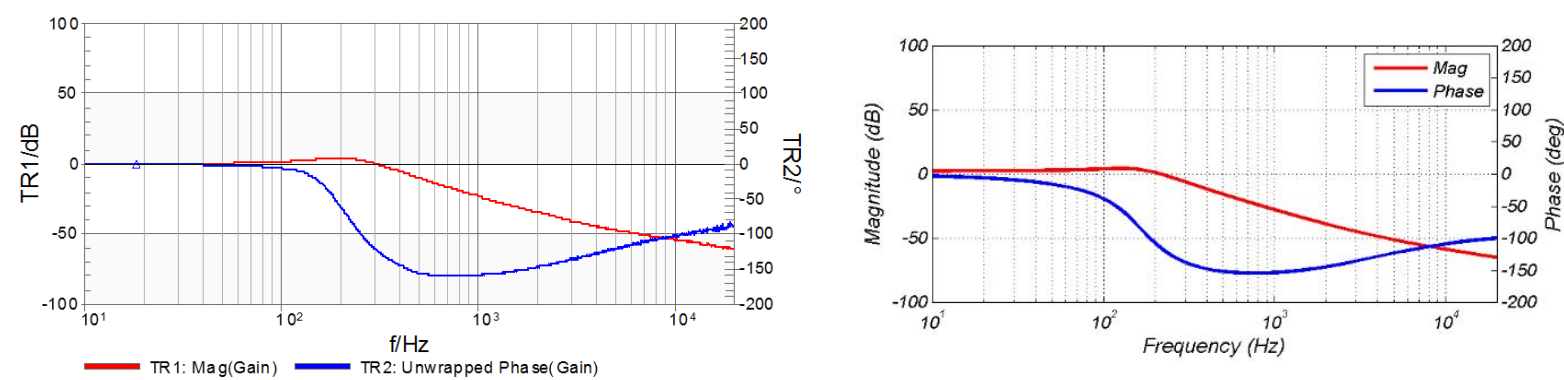

(a)
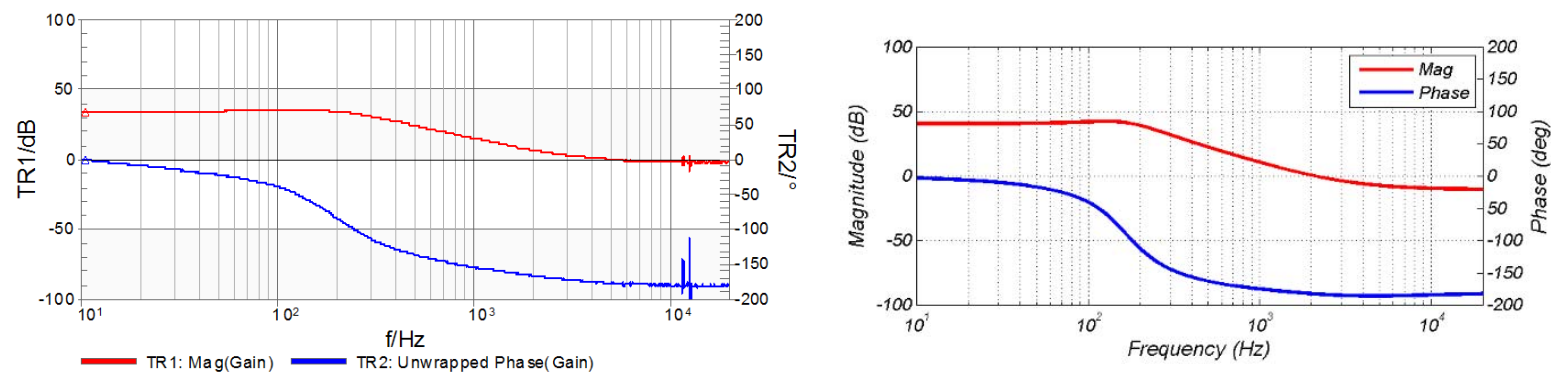

(b)

Fig. 9. Experimentally obtained frequency responses (left column) and corresponding simulated results (right column) for (a) the input to capacitor voltage $\left(G_{v v}\right)$ and (b) control to capacitor voltage $\left(G_{v d}\right)$ transfer functions of PWM Y-source converter.

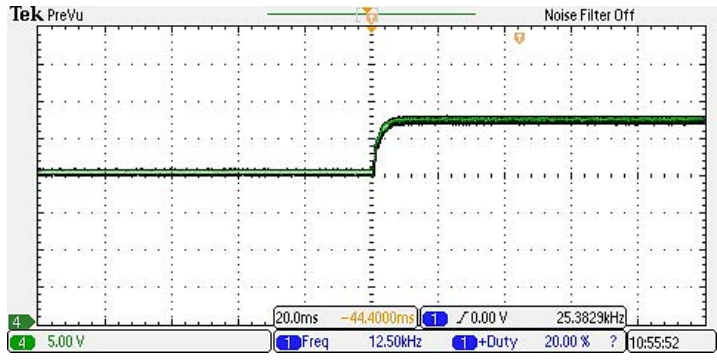

(a)

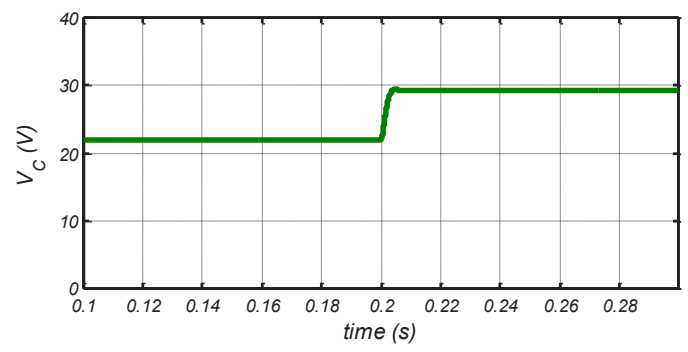

(b)

Fig. 10. Dynamic response of PWM Y-source dc-dc converter to a step change in input voltage from $15 \mathrm{~V}$ to 20 $\mathrm{V}$, (a) experimentally obtained and (b) theoretically predicted.

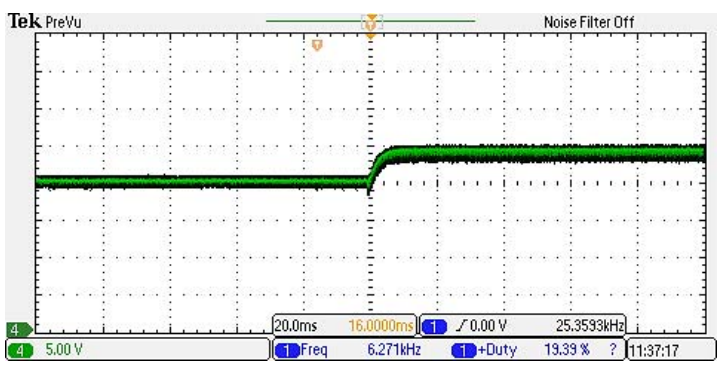

(a)

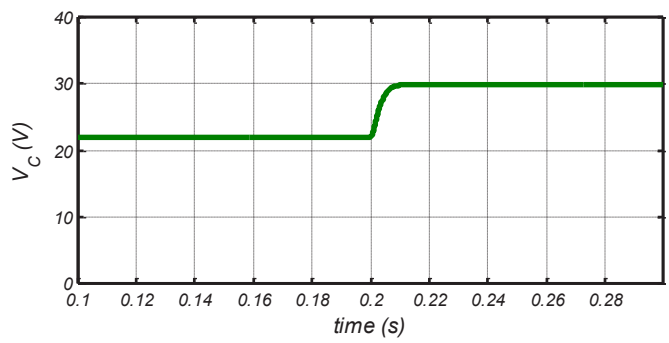

(b)

Fig. 11. Dynamic response of PWM Y-source dc-dc converter to a step change in duty cycle from 0.1 to 0.15 , (a) experimentally obtained and (b) theoretically predicted. 


\section{Conclusion}

Small signal modeling of the PWM Y-source converter by means of circuit averaging and averaged switch technique has been presented in this paper. Dc averaged model and ac small signal model of the PWM Y-source converter have been demonstrated. Besides, the input voltage to capacitor voltage and control to capacitor voltage transfer functions have been derived considering perturbations in input voltage and duty cycle, respectively. The derived transfer functions have been validated through simulation results using MATLAB/Simulink software. It has been shown that the predicted results from the transfer functions are in good agreement with the frequency response from detailed circuit in Simulink. Finally, the effectiveness of the derived model has been validated through experimental results of the frequency and dynamic responses of PWM Y-source converter.

\section{References}

[1] Y. P. Siwakoti, F. Z. Peng, F. Blaabjerg, P. C. Loh, and G. E. Town, "Impedance-Source Networks for Electric Power Conversion Part I: A Topological Review," IEEE Trans. Power Electron., vol. 30, no. 2, pp. 699-716, Feb. 2015.

[2] R. Strzelecki, M. Adamowicz, N. Strzelecka, and W. Bury, "New type T-source inverter," in Proc. CPE, Badajoz, 2009, pp. 191-195.

[3] W. Qian, F. Z. Peng, and H. Cha, "Trans-Z-source Inverters," IEEE Trans. Power Electron., vol. 26, no. 12, pp. 3453-3463, Dec. 2011.

[4] P. C. Loh, D. Li, and F. Blaabjerg, "Г-Z-source Inverters," IEEE Trans. Power Electron. (letters), vol. 28, no. 11, pp. 4880-4884, Nov. 2013.

[5] Y. P. Siwakoti, P. C. Loh, F. Blaabjerg and G. E. Town, "Y-Source Impedance Network," IEEE Trans. Power Electron. (letters), vol. 29, no. 7, pp. 3250-3254, July 2014.

[6] Y. P. Siwakoti, F. Blaabjerg and P. C. Loh, "Quasi Y-source boost DC-DC converter," in Proc. EPE'15 ECCE-Europe, Geneva, 2015, pp. 1-10.

[7] M. Forouzesh and A. Baghramian, "Galvanically isolated high gain Y-source DC-DC converters for dispersed power generation," IET Power Electron., vol. 9, no. 6, pp. 1192-1203, 2016.

[8] M. Forouzesh, N. Salavati and A. Baghramian, "Improved Y-source inverter for distributed power generation," in Proc. 2015 23rd ICEE, Tehran, 2015, pp. 1677-1681.

[9] Y. P. Siwakoti, F. Z. Peng, F. Blaabjerg, P. C. Loh, G. E. Town, and S. Yang, "Impedance-Source Networks for Electric Power Conversion Part II: Review of Control and Modulation Techniques," IEEE Trans. Power Electron., vol. 30, no. 4, pp. 1887-1906, April 2015.

[10] C. J. Gajanayake, D. M. Vilathgamuwa, and P. C. Loh, "Small-Signal and Signal-Flow-Graph Modeling of Switched Z-source Impedance Network," IEEE Trans. Power Electron. Letters, vol. 3, no. 3, pp. 111-116, Sep. 2005.

[11] J. Liu, J. Hu, and L. Xu, "Dynamic Modeling and Analysis of Z-source Converter-Derivation of AC Small Signal Model and Design-Oriented Analysis," IEEE Trans. Power Electron., vol. 22, no. 5, pp. 17861796, Sep. 2007.

[12] Y. Li, and F. Z. Peng, "AC small signal Modeling, analysis and control of quasi-Z-Source Converter," in Proc. IPEMC, Harbin 2012, pp. 1848-1854.

[13] V. P. Galigekere, and M. K. Kazimierczuk, "Small-signal modeling of PWM Z-source converter by circuitaveraging technique," in Proc. ISCAS, Rio de Janeiro, 2011, pp. 1600-1603.

[14] R. W. Erickson, and D. Maksimovic, Fundamentals of power electronics: Springer Science \& Business Media, 2007.

[15] M. K. Kazimierczuk, Pulse-width modulated DC-DC power converters: John Wiley \& Sons, 2008.

[16] S. Skogestad, and I. Postlethwaite, Multivariable feedback control: analysis and design vol. 2: Wiley New York, 2007. 